\title{
Evaluating the Effect of Leg Position and Negative Pressure Wound Therapy on Lower Extremity Oxygenation Measured by Noninvasive Tissue Oximetry: A Pilot Study
}

\author{
Claire Isabelle Verret, BS ${ }^{1,2}$ Meridith Pollie, BS $^{1,2} \quad$ Jona Kerluku, BS ${ }^{1}$ Sravan Dhulipala, MD ${ }^{1}$ \\ Duretti Fufa, MD ${ }^{1,2}$ \\ ${ }^{1}$ Hospital for Special Surgery, New York, New York \\ 2 Weill Cornell Medical College, New York, New York \\ Address for correspondence Claire I. Verret, BS, Hospital for Special \\ Surgery, 535 E 70th street, New York, New York 10021 \\ J Reconstr Microsurg Open 2020;5:e79-e81. \\ (e-mail: civ2002@med.cornell.edu; cisabellecverret@yahoo.com).
}

\begin{abstract}
Background Flap loss is a catastrophic complication following lower extremity reconstructions. Close monitoring can detect circulatory changes, particularly when introducing gravity through dangle protocols. Noninvasive near-infrared spectroscopy (NIRS) tissue oximetry is one such method used for continuous monitoring of tissue perfusion. Recently, negative pressure wound therapy (NPWT) has been for compromised flaps salvage. We used NIRS to study the impact of leg positioning and NPWT on lower extremity perfusion during a mock dangle protocol.

Methods A tissue oximeter transcutaneous probe was placed on each lower limb of five normal patients at the location of the highest density of peroneal artery perforators along the distal one-third fibula. The experimental leg probe was covered with NPWT at $100 \mathrm{~mm} \mathrm{Hg}$. The contralateral leg served as control. Tissue oxygen saturation $\left(\mathrm{StO}_{2}\right)$ was measured continuously for 140 minutes. Leg position was

\section{Keywords}

- negative pressure wound therapy

- noninvasive tissue oximetry

- lower extremity oxygenation

changed in every 20 minutes to mimic a dangle protocol.

Results Our results showed higher $\mathrm{StO}_{2}$ with leg elevation, and lower $\mathrm{StO}_{2}$ in dependent positions in the control limb. $\mathrm{StO}_{2}$ was consistently higher in the limb with NPWT, which had a slower rate of deoxygenation with introduction of gravity. In both conditions, on returning to neutral position from the dependent, $\mathrm{StO}_{2}$ returned to levels close to baseline.

Conclusion These data provide further information on hemodynamic changes introduced by gravity, and how NPWT might modulate these changes.
\end{abstract}

Lower extremity free flaps have higher rates than other anatomical areas due to gravitational challenges associated with lower limb dependency. ${ }^{1}$ Structured protocols and close monitoring are key to gradually habituate newly transferred tissues to microcirculatory changes, ${ }^{1}$ particularly when introducing gravity through dangle protocols.

While clinical examination is the gold standard, newer technologies, including near infrared spectroscopy (NIRS) tissue oximetry, are used as adjuncts to observation. ${ }^{2}$ NIRS

received

May 3, 2020

accepted

August 15, 2020
DOI https://doi.org/

10.1055/s-0040-1717110. ISSN 2377-0813. continuously tracks tissue oxygenation, allowing for detection of falling oxygen saturation and identification of venous thromboses before clinically detectable.

Meanwhile, negative pressure wound therapy (NPWT) is currently being explored to improve circulation in early compromised flaps. Though designed for open wound management, NPWT has been reported as a safe method for possible flap salvage. ${ }^{3}$ Little is, however, known about the specific effects of NPWT on circulation. Because NIRS
Copyright $\odot 2020$ by Thieme Medical Publishers, Inc., 333 Seventh Avenue, New York, NY 10001, USA. Tel: +1(212) 760-0888.
License terms

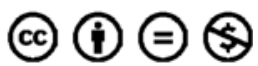


provides minute-to-minute information regarding tissue oxygenation, its potential uses include detecting circulatory changes with varying lower limb positions, and determining the impact of NPWT on the rate and/or magnitude of these changes.

The purpose of this pilot study was to (1) use NIRS to assess the effect of leg positioning on tissue oxygenation in the lower extremity in various positions of dependency modeling a dangle protocol, and (2) assess the impact of NPWT on tissue oxygenation throughout these positional changes.

\section{Methods}

A transcutaneous Vioptix ODISsey Tissue Oximeter (ViOptix Inc., Fremont, CA) probe was placed on each lower limb of a healthy, normal patient, in the location of the highest density of peroneal artery perforators along the distal one-third fibula. The experimental leg probe was covered with a NPWT device set to $100 \mathrm{~mm} \mathrm{Hg}$. The contralateral leg probe remained uncovered, serving as control. Tissue oxygen saturation $\left(\mathrm{StO}_{2}\right)$ was measured continuously for 140 minutes; percent $\mathrm{StO}_{2}$ was recoded in every 2 minutes. Bilateral leg position was changed in every 20 minutes emulating this dangle protocol: supine 1 (heart level), elevated $(30 \mathrm{~cm})$, supine 2 , seated 1 (45-degree dangle), standing, seated 2 , and supine 3 positions. Reported $\mathrm{StO}_{2}$ for each leg position is the average of the 10 values recorded.

\section{Results}

In the control limb (without NPWT), $\mathrm{StO}_{2}$ increased from supine $(74.3 \%)$ to elevation (76.9\%), and then decreased (73.9\%) upon return to supine (-Table 1). With introduction of gravity, $\mathrm{StO}_{2}$ decreased, reaching its lowest value (52.8\%) 16 minutes into seated 1 posture (-Fig. 1). The saturation further decreased during standing and seated 2 positions. Upon final return to supine, average $\mathrm{StO}_{2}$ increased to $71.2 \%$.

In the experimental limb (with NPWT), positional trends in $\mathrm{StO}_{2}$ were similar to the control. $\mathrm{StO}_{2}$ was, however, consistently higher during all positions compared with the contralateral limb by an average of $4.7 \%$. $\mathrm{StO}_{2}$ remained stable during nondependent positions: supine 1 (79.2\%), elevation (78.3\%), and supine 2 (79.8\%). With introduction of gravity, $\mathrm{StO}_{2}$ progressively decreased from seated $1(71.7 \%)$ to standing (65.7\%) to seated 2 (62.2\%) positions; reaching its lowest value
(60.6\%) in 50 minutes during seated 2 position ( - Fig. 1). Upon return to supine, $\mathrm{StO}_{2}$ increased to $75.6 \%$.

\section{Discussion}

This pilot study showed $2.6 \%$ increase in $\mathrm{StO}_{2}$ with leg elevation, and $19.8 \%$ decrease with dependency in the control limb. With application of NPWT, $\mathrm{StO}_{2}$ was higher compared with control, throughout all limb positions, by an average $4.7 \%$. The gravity-related decrease in $\mathrm{StO}_{2}$ was more gradual in the NPWT limb compared with the control ( 50 vs. 16 minutes). In both test conditions, $\mathrm{StO}_{2}$ recovered to levels close to baseline after dangle.

In both limbs, $\mathrm{StO}_{2}$ increased or remained stable with elevation, decreased with dependency, reaching lowest average value during seated 1 and 2 positions, followed by rapid increase in $\mathrm{StO}_{2}$ levels upon return to supine. These observations are consistent with previous studies. ${ }^{1,4}$ Desaturation associated with dependency is likely caused by venous pooling, which diminishes arterial blood flow to the affected area and over time decreases oxygen saturation. ${ }^{1}$

Our results validate the observations by Kolbenschlag et al that sustained pressure application on lower extremity free flaps $(30 \mathrm{~mm} \mathrm{Hg}$ controlled-pressure elastic compression wrapping) significantly improved tissue oxygenation during dangling in the postoperative period. ${ }^{1}$ They concluded that increased pressure improved venous drainage, thus increasing blood flow and preventing oxygen desaturation. In our study, higher average $\mathrm{StO}_{2}$ was observed with the addition of NPWT at $100 \mathrm{~mm} \mathrm{Hg}$ during dependency compared with the control limb. However, while these authors reported higher $\mathrm{StO}_{2}$ in the control leg versus the compressed leg in neutral position, we found increased $\mathrm{StO}_{2}$ in the NPWT limb while supine. This difference may be due to a more focused pressure applied by the NPWT device compared with the compressive wrap, resulting in more blood flow to the leg. Additionally, perfusion differences with limited venous pooling in healthy legs compared with those following free flap reconstruction may have also contributed to this finding. The smallest difference between NPWT and control limbs was noted in the elevated position. This further supports this impact of NPWT on venous pooling, which is least relevant in the elevated position.

The most notable finding was the slower rate of dependent desaturation with NPWT. While the control limb

Table 1 Effects of leg position and use of NPWT on lower extremity $\% \mathrm{StO}_{2}$

\begin{tabular}{|c|c|c|c|c|c|c|c|c|}
\hline $\begin{array}{l}\text { Limb position } \\
\text { average } \\
\mathrm{StO}_{2}\end{array}$ & $\begin{array}{l}1 \\
\text { Supine } 1\end{array}$ & $\begin{array}{l}2 \\
\text { Elevated }\end{array}$ & $\begin{array}{l}3 \\
\text { Supine } 2\end{array}$ & $\begin{array}{l}4 \\
\text { Seated } 1\end{array}$ & $\begin{array}{l}5 \\
\text { Standing }\end{array}$ & $\begin{array}{l}6 \\
\text { Seated } 2\end{array}$ & $\begin{array}{l}7 \\
\text { Supine } 3\end{array}$ & Average \\
\hline Control (\%) & 74.3 & 76.9 & 73.9 & 57.1 & 55.5 & 54.1 & 71.2 & - \\
\hline Experimental (\%) & 79.2 & 78.3 & 79.8 & 71.7 & 65.7 & 62.2 & 75.6 & - \\
\hline$\Delta(\%)$ & 4.9 & 1.3 & 5.9 & 14.6 & 10.2 & 8.1 & 4.5 & 4.7 \\
\hline
\end{tabular}

Abbreviations: NPWT, negative pressure wound therapy; $\% \mathrm{StO}_{2}$, tissue oxygen saturation

Notes: Control: without NPWT; experimental: with NPWT.

$\Delta$ : experimental $\% \mathrm{StO}_{2}-$ control $\% \mathrm{StO}_{2}$ 


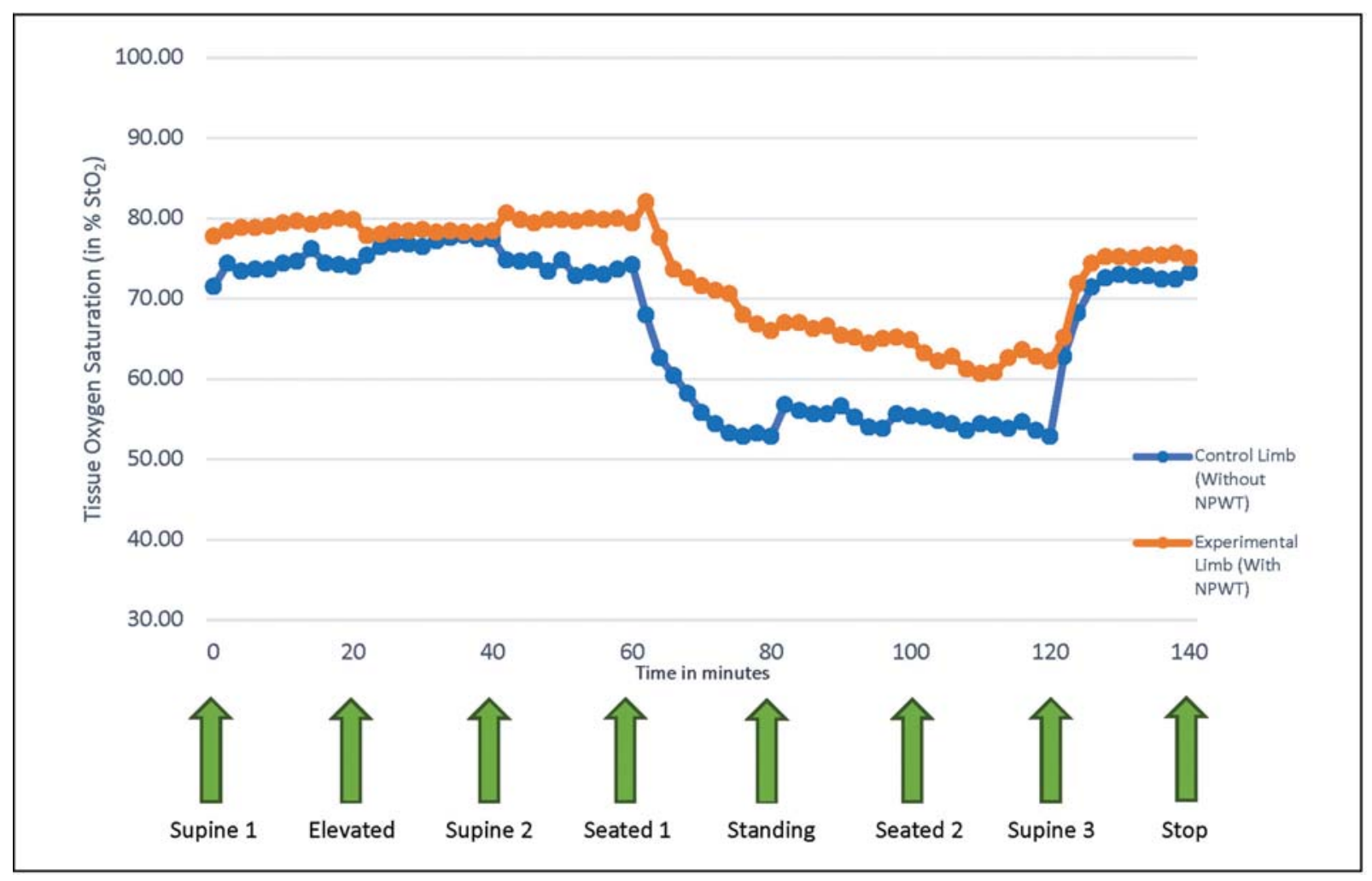

Fig. 1 Lower extremity tissue oxygen saturation, limb position and use of negative pressure wound therapy.

reached its nadir within 16 minutes of dependency (seated 1 position), the NPWT limb did not reach its nadir until 50 minutes of dependency (seated 2 position). This finding may be clinically relevant, supporting the use of NPWT as a potential tool to decrease the impact of gravity following lower limb flap reconstruction.

These results demonstrate that microcirculatory changes related to tissue oxygenation depend on limb position, and can be measured with NIRS. The addition of NPWT may increase tissue oxygenation in all limb positions and slow the rate of deoxygenation with introduction of gravity, and may be comparable to elastic compression wrapping. ${ }^{5}$

\section{Limitations and Conclusion}

This pilot study is limited in its performance in a small sample of normal limbs instead of limbs following free flap reconstruction. The sensitive nature of the immediate postoperative period following free flap reconstruction would not have allowed such a structured observation of the impact of prolonged gravity in postoperative patients. Due to the small sample size, statistical significance cannot be assessed. However, these results may be used to generate hypotheses testable in clinical settings. Despite these limitations, these data provide additional information and insight on hemodynamic changes introduced by gravity, and how NPWT may modulate these changes.

\section{Conflict of Interest}

None declared.

\section{References}

1 Kolbenschlag J, Ruikis A, Faulhaber L, et al. Elastic wrapping of lower extremity free flaps during dangling improves microcirculation and reduces pain as well as edema. J Reconstr Microsurg 2019;35(07):522-528

2 Keller A. Noninvasive tissue oximetry. Clin Plast Surg 2011;38 (02):313-324

3 Bi H, Khan M, Li J, Pestana IA. Use of incisional negative pressure wound therapy in skin-containing free tissue transfer. J Reconstr Microsurg 2018;34(03):200-205

4 Kolbenschlag J, Bredenbroeker P, Daigeler A, et al. Changes of oxygenation and hemoglobin-concentration in lower extremity free flaps during dangling. J Reconstr Microsurg 2014;30(05): 319-328

5 Sogorski A, Lehnhardt M, Goertz O, et al. Improvement of local microcirculation through intermittent negative pressure wound therapy (NPWT). J Tissue Viability 2018;27(04):267-273 